\title{
Pulmonary metastasectomy in colorectal cancer: a randomized controlled trial
}

\section{Dear Editor,}

A preprint of pulmonary metastasectomy in colorectal cancer (PulMiCC) was included in the meta-analysis of pulmonary metastasectomy in patients with colorectal cancer by Ratnayake et al. ${ }^{1}$ The PulMiCC trial was published in full with 93 patients randomized either to undergo surgical metastasectomy or not. There was no crossover from the control to the treatment arm and no difference in survival (hazard ratio $0.93,95 \%$ confidence interval 0.56 1.56). (Figure 1) Although not significant, median and 4-year survival favoured the control group over the surgical group at 3.8 versus 3.5 years, and $47 \%$ versus $44 \%$, respectively. ${ }^{2}$ There was no quality of life or health utility benefit. ${ }^{3}$ We are puzzled by the comment that there was 'an unclear risk of selection bias in the failure to report a further detail on the participant population'. There was no difference in seven important prognostic factors between the two arms, which were well balanced.

We doubt that it is appropriate or meaningful to include the PulMiCC Randomised Controlled Trial in a meta-analysis with two non-randomized studies (Ratnayake et al., ${ }^{1}$ figs 2c,d). Comparisons of survival using data from retrospective cohort studies (as all the other nine studies in this meta-analysis appear to be) are unreliable. They will inevitably be confounded by selection bias from known

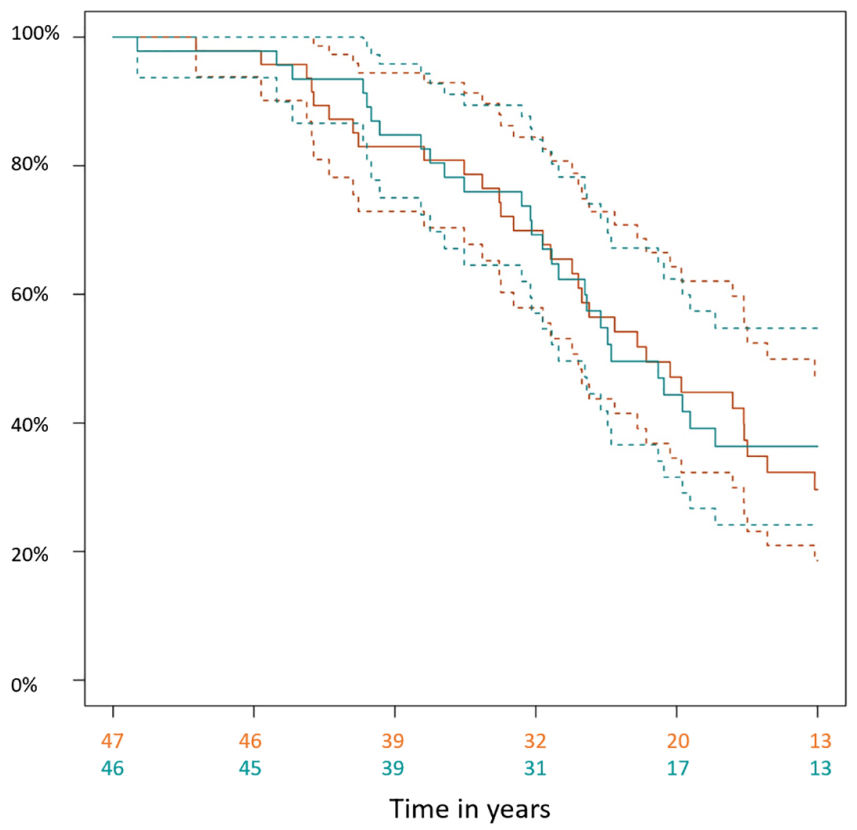

Fig 1. The Kaplan-Meier analysis of the pulmonary metastasectomy in colorectal cancer trial. (—) Control; (-) metastasectomy.

and unknown prognostic factors. This is obvious in the Methodological Index for NOn-Randomised Studies scores (Ratnayake et al., ${ }^{1}$ supplementary table 4), which shows that six studies scored 0 , and three 1 , for criterion 11 - 'equivalent baseline characteristics'.

PulMiCC findings indicate that only large-scale phase III randomized trials can now determine whether there is any benefit from metastasectomy. What is clear from the PulMiCC trial is that Society of Thoracic Surgeons consensus assumed 'zero' 5-year survival is untrue. ${ }^{4}$ The more usual estimate of $<5 \%$ would have been replicated if there were only $2 / 47(4.2 \%)$ control survivors whereas there were $13 / 47(27.6 \%), P=0.0036$ by Fisher's exact test.

\section{Author Contributions}

Fergus Macbeth: Conceptualization; data curation; methodology; project administration; supervision; writing-original draft. Norman Williams: Data curation; formal analysis; methodology; project administration; software; validation; writing-review \& editing.

\section{References}

1. Ratnayake CBB, Wells CI, Atherton P et al. Meta-analysis of survival outcomes following surgical and non surgical treatments for colorectal cancer metastasis to the lung. ANZ J. Surg. 2020. https://doi.org/10. 1111/ans.16383.

2. Milosevic M, Edwards J, Tsang D et al. Pulmonary metastasectomy in colorectal cancer: updated analysis of 93 randomized patients - control survival is much better than previously assumed. Colorectal Dis. 2020; 22: 1314-24.

3. Brew-Graves C, Farewell V, Monson $\mathrm{K}$ et al. Pulmonary metastasectomy in colorectal cancer: health utility scores by EQ-5D-3L in a randomised controlled trial show no benefit from lung metastasectomy. Colorectal Dis. 2021; 23(1): 200-5.

4. Handy JR, Bremner RM, Crocenzi TS et al. Expert consensus document on pulmonary metastasectomy. Ann. Thorac. Surg. 2019; 107: 631-49.

Fergus Macbeth,* DM, FRCP Norman R. Williams, $\dagger \mathrm{PhD}$ Tom Treasure, $\$$ MD, MS, FRCS, FRCP (D) *Centre for Trials Research, Cardiff University, Cardiff, UK, $\uparrow$ Surgical and Interventional Trials Unit, University College London, London, UK and $\$$ Clinical Operational Research Unit, University College London, London, UK

doi: 10.1111/ans.16658 